\title{
Complex patterns of male germline instability and somatic mosaicism in myotonic dystrophy type 1
}

\author{
Loreto Martorell ${ }^{1}$, Darren G Monckton ${ }^{2}$, José Gamez ${ }^{3}$ and Montserrat Baiget ${ }^{1}$
}

${ }^{1}$ Servei de Genètica, Hospital de Sant Pau, Barcelona, Spain; ${ }^{2}$ Division of Molecular Genetics, Institute of Biomedical
and Life Sciences, University of Glasgow, UK; ${ }^{3}$ Servei de Neurología, Hospital de la Vall d'Hebrón, Barcelona, Spain

The genetic basis of myotonic dystrophy type 1 (DM1) is the expansion of a CTG repeat in the 3' untranslated region of DMIPK. Once into the disease range, the repeat becomes highly unstable and is biased toward expansion in both somatic and germline tissues. Intergenerational differences usually reveal an increase in allele length, concordant with the clinical anticipation characteristic of DM 1, but there have also been cases with intergenerational contractions of the repeat length, accompanied by apparent anticipation. In order to gain a better understanding of this intergenerational behaviour, we have obtained semen samples from six DM males and used single molecule analyses to compare the allele distributions present in their sperm and blood with those of their offspring. We have confirmed that the male germline mutational pathway is distinct from that of the soma, but the extent of variation is highly variable from one individual to another and not obviously correlated with progenitor allele length. Nonetheless, in all cases the alleles present in the father's sperm overlap with those observed in their offspring. These data also provide further indications that the interpretation of intergenerational transmissions by standard analyses is frequently compromised by the masking of germline differences by age-dependent somatic expansion in the parent. European Journal of Human Genetics (2000) 8, 423-430.

Keywords: germline; CTG repeat; instability; mosaicism; contraction

\begin{abstract}
Introduction
Myotonic dystrophy (DM) is an extremely variable autosomal dominant multisystemic disorder, with myotonia, muscle weakness and wasting, heart abnormalities, cataracts and testicular atrophy as common features. ${ }^{1}$ Indeed, DM is the most common form of adult onset muscular dystrophy with an estimated world-wide incidence of around 1 in 8000 individuals. Three main clinical forms have been described according to the age of onset and severity of symptoms: mild form with cataracts and sometimes very mild muscle-based symptoms with onset in old age; classic adult onset form with a clear implication of muscular and other systems appearing in young and adult patients; and the congenital form, that is the most severe form of the disease where the symptoms are detectable at birth. Congenital DM (CDM) babies are born with extreme hypotonia and frequently diein the neonatal period due to respiratory distress.
\end{abstract}

Correspondence: Loreto Martorell, Servei de Genètica, Hospital de Sant Pau, Pare Claret 167, 08025-Barcelona, Spain. Tel: +34 32919194 ; Fax: +34 3 2919494; E-mail: genm@santpau.es

Received 15 November 1999; revised 5 January 2000; accepted 20 January 2000
The vast majority of DM families are associated with the expansion of a CTG repeat in the $3^{\prime}$ untranslated region of a protein kinase gene (DMIPK)., ${ }^{2,3}$ This classical form of the disease is now termed myotonic dystrophy type 1 (DM1) (as recommended by the participants of the 2nd International Myotonic Dystrophy Consortium meeting and approved by the Nomenclature Committee of HUGO). A subset of families does not have the DM IPK CTG expansion and is associated with either a second locus on chromosome $3 q$ (DM2) or remains unmapped..$^{4,5}$

The number of CTG repeats at this locus within the general population has been shown to be highly polymorphic. Intergenerational expansion of the repeat length is seen in DM 1 families and underlies the phenomenon of anticipation, ${ }^{6}$ that is an increasing severity and decreasing age of onset in successive generations of a family. M olecular studies have suggested sex-related differences in the intergenerational amplification of the DM 1 gene. Small expansions are most unstable when transmitted by males, giving rise to the excess of transmitting grandfathers in DM1 pedigrees, ${ }^{6,7}$ whilst the largest expansions associated with CDM 1 are usually only transmitted by females. ${ }^{8,9}$ 
Intergenerational DM 1 transmissions have almost exclusively been determined by measuring the blood allele size in parent and offspring. Whilst in the main such observations usually correlate quite well with the observed clinical anticipation, quite a high proportion of cases have been demonstrated apparent reductions in the allele length not associated with an improvement of the patients clinical symptoms. ${ }^{10}$ Since it is known that the expanded CTG repeat is highly unstable in both somatic tissues ${ }^{11,12}$ and in the male germline, ${ }^{13,14}$ some account of these factors needs to be made in interpreting measured intergenerational differences, given that the somatic changes in repeat length could influence the interpretation of intergenerational changes. Detailed studies of somatic mosaicism have revealed that it is tissue specific, ${ }^{11-13}$ biased toward further expansion and continuous throughout the life of an individual. ${ }^{14-17}$ Clearly, progressive somatic mosaicism has implications for interpreting intergenerational transmissions based on the analysis of average allele sizes in the blood of parent and offspring. In such situations parent and child have most likely been sampled on the same date, at which point their ages are likely to differ in the order of 20 to 40 years. Without some account taken of the age effect it is possible that the measured differences in average blood allele sizes will not reflect the true germline change. In order to gain a better understanding of the dynamics of repeat instability in the male germline and the basis of measured intergenerational differences, we have used sensitive small pool-PCR analyses (SP-PCR) ${ }^{14,18}$ to compare blood and sperm DNA from six transmitting DM males with DNA samples from their offspring.

\section{Materials and methods Patients samples}

Samples were obtained from Spanish DM 1 patients who were referred to the Genetics Unit at the Hospital de Sant Pau, Barcelona, for diagnostic testing. Informed consent was obtained from all families after the nature and possible consequences of the study had been explained. Genomic DNA was prepared from peripheral blood leukocytes using standard procedures. ${ }^{19}$ Semen obtained from six DM1 patients was diluted with an equal volume of $1 \times$ SSC $(0.15 \mathrm{M}$ $\mathrm{NaCl}, 15 \mathrm{~mm}$ Trisodium citrate, $\mathrm{pH} 7.0$ ), 1\% SDS to lyse epithelial cells and seminal leukocytes. Sperm were collected by centrifugation at $10000 \mathrm{~g}$ for $5 \mathrm{~min}$. The cell pellet was rinsed two times in $1 \mathrm{ml}$ of $1 \times \mathrm{SSC}, 1 \% \mathrm{SDS}$ and then resuspended and incubated in $500 \mathrm{mgr} ; \mathrm{l}$ of $3.0 \mathrm{M} \mathrm{NaCl}, 20 \mathrm{~mm}$ EDTA, $0.01 \mathrm{~m}$ Tris- $\mathrm{HCl}(\mathrm{pH} 8.0), 2 \% \mathrm{SDS}$ and $1 \mathrm{mgml}^{-1}$ proteinase $\mathrm{K}$ at $37^{\circ} \mathrm{C}$ overnight. Sperm DNA was collected after phenol extraction by ethanol precipitation.

\section{Determination of CTG repeat size}

The size of the CTG repeat expansion was analysed by PCR and/or Southern blotting. Large expansions were detected by Southern blot analysis: $5 \mu \mathrm{g}$ of genomic DNA was digested with EcoRl or Bgll (Gibco-BRL Life Technologies SA, BarceIona, Spain) and electrophoresed on 0.6 t $0.8 \%$ agarose gels, denatured in $0.5 \mathrm{M} \mathrm{NaOH}, 1 \mathrm{M} \mathrm{NaCl}$, neutralised in $0.5 \mathrm{M}$ Tris$\mathrm{HCl}(\mathrm{pH} 7.0), 1 \mathrm{M} \mathrm{NaCl}$ and transferred on to Hybond- $\mathrm{N}$ membrane in $10 \times$ SSC (Amersham Pharmacia Biotech SA, Cerdanyola, Barcelona, Spain). Filters were probed with CDNA25, labelled with ${ }^{32} \mathrm{P}$ using the random priming method. Blots were washed at $0.2 \times$ SSC, $0.2 \%$ SDS final stringency at $65^{\circ} \mathrm{C}$, and were exposed for $5-10$ days at $-80^{\circ} \mathrm{C}$. The modal size of the expansion was determined as the point of highest band intensity on the autoradiograph or at the centre of the smear for very diffuse bands. Parent and offspring samples were compared side by side on the same gel. PCR was used to determine the number of CTG repeats in normal alleles and small repeat amplifications ( $<200$ repeats) in DM 1 patients. PCR reactions were carried out in a total volume of $50 \mu$ l using 700ng of genomic DNA. Radiolabel was added to the PCR samples for detection of products on polyacrylamide gels using the previously described conditions. ${ }^{20}$ The allele lengths were assessed by comparison of the electrophoretic migration pattern of the PCR product in the gel with an $\mathrm{M}-13$ sequence ladder used as a size marker (Sequenase Kit, Amersham Pharmacia Biotech SA, Cerdanyola, Barcelona, Spain).

\section{Small pool PCR}

Small pool-PCR (SP-PCR) analysis was performed to resolve heterogeneous smears on DM 1 repeat length distributions into their component parts essentially as previously described ${ }^{14}$ with slight modifications: genomic DNA samples were digested with EcoRI and diluted in $10 \mathrm{~mm}$ Tris- $\mathrm{HCl}$ $(\mathrm{pH} 7.5), 1 \mathrm{~mm}$ EDTA and $0.1 \mu \mathrm{m}$ carrier primer DM-A. 3-300 pg pools of EcoRI (Gibco-BRL) digested DNA were amplified in $7 \mu \mathrm{l}$ reactions (PE Applied Biosystems, Foster

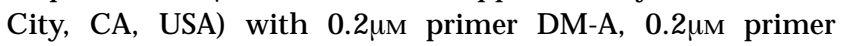
DM-BR and $0.05 \mathrm{Uml}^{-1}$ of Taq DNA polymerase (Roche Diagnostics SL, Barcelona, Spain). $5 \mu$ l of the reaction product were loaded on $1.5-2 \%$ agarose gels and electrophoresis was performed at $90 \mathrm{~V}$ for $16 \mathrm{~h}$. The products were analysed by Southern blotting with subsequent hybridisation to a radiol abelled (CTG) ${ }_{10}$ oligonucleotide probe. For each set of PCR amplifications we also set up multiple zero DNA control reactions using equivalent dilutions of EcoRI digest with no DNA added.

\section{Results}

In order to gain a greater understanding of the individual specific dynamics of intergenerational transmissions in DM 1 families we obtained semen samples from six DM 1 males who had either the classic adult onset form of the disease or were currently asymptomatic. All also had either fathered children with expansions or had prenatal diagnoses performed on carrier foetuses (Figure1). A simple analysis of the intergenerational length change was performed using the 
FAMILY-I

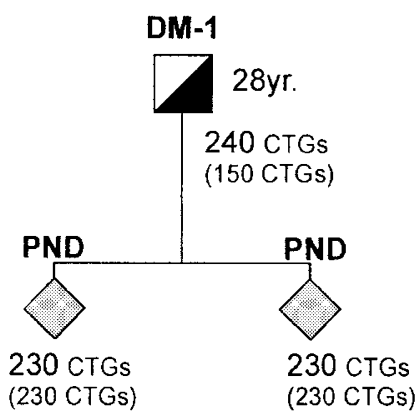

FAMILY-III

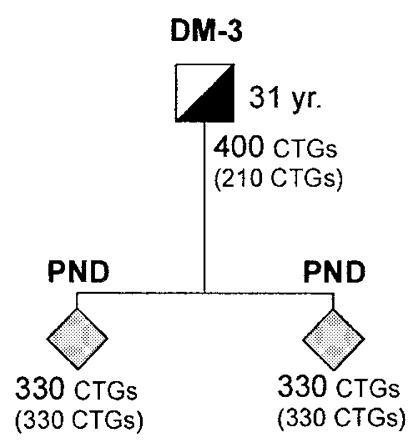

FAMILY-V

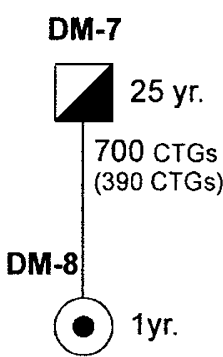

250 CTGs (250 CTGs)

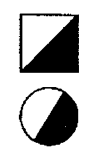
CLASSICAL

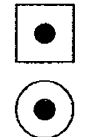

MILD OR ASYMPTOMATIC
FAMILY-II

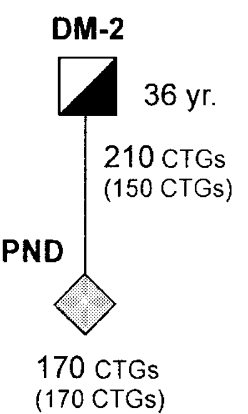

FAMILY-IV

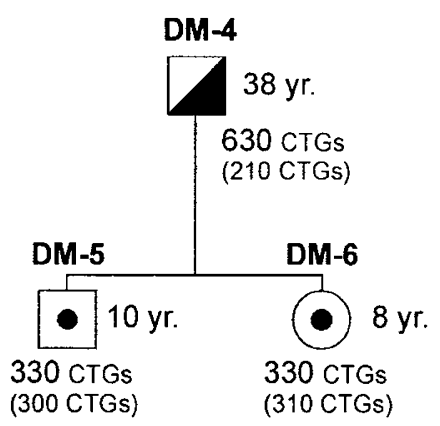

FAMILY-VI

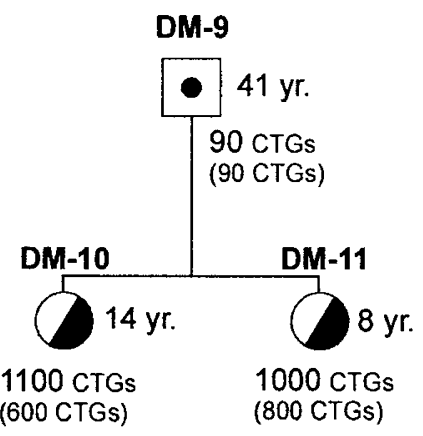

Figure 1 Intergenerational transmission in DM1 families. Shown are the pedigrees of the six DM1 families studied. Shown for each individual is their identification number (DM-X), age at sampling (yr. =years, PND = prenatal diagnosis) and approximate average expanded blood allele size as determined by standard restriction digested genomic DNA Southern blot analyses. The estimated size of the progenitor allele is indicated in parenthesis. 
blood DNA of the father and compared with the DNA of their children and/or carrier foetuses using standard restriction digested genomic DNA Southern blot analyses. In a more detailed analyses of these families sperm DNA was isolated from the semen samples and the allele distributions present in the male germline determined using sensitive single molecule SP-PCR procedures (Table1, Figures2 and 3). The sperm distributions were compared directly with their blood and those of the blood DNA of their offspring also using SP-PCR (Tables 1 and 2, Figures 2 and 3). Both blood and sperm DNA from the fathers demonstrated high levels of mosaicism for the expanded allele in all of the individuals, with the exception of individual DM-9. The blood DNA distributions for those males (DM-1, $-2,-3$ and -9) with the smaller alleles demonstrated positively skewed distributions typical of smaller expanded DM alleles, whilst the two males (DM-4 and -7) with the largest expansions demonstrated even higher levels of diversity with more normal two-tailed distributions. All of the sperm samples showed a wide range of diverse alleles with more normal two-tailed distributions, all including a lower tail that exten ded down toward the high end of the normal size range. The largest germline expansion is detected in the patient that carries the shortest progenitor allele and shows relatively little somatic instability. In every case the allele size distribution of the children or foetuses were consistent with an allele still present in the father's sperm. In familiesI, II and III prenatal diagnosis using standard procedures predicted that the carrier foetuses had inherited either zero length change expanded alleles, or in one case (familyli) a small intergenerational contraction. DM-4 has fathered two children in familyIV both with expansions of about 330 repeats, apparent intergenerational contractions, and both of whom currently remain asymptomatic (aged 10, DM-5 and 8 years, DM-6). Male DM-7 from family $V$ had a relatively large expanded allele of average size, about 700 repeats, as detected in his blood DNA by standard Southern blot analyses. He has fathered one daughter who inherited the mutant DM chromosome with an expanded allele of average size of about 250 repeats, and at 1 year old she remains asymptomatic (DM-8). The transmitting male in family VI, DM-9, was an asymptomatic 41-year-old, concordant with his age and his relatively small expansion size of about 90 repeats. However, he has fathered two daughters with very large expansions (average allele sizes of 1100 repeats aged $14, \mathrm{DM}-10$, and about 1000 repeats aged 8 , DM-11) and both have a severe juvenile onset form of the disease, including moderate mental retardation (Figure1).

\section{Discussion}

The expanded repeat in DM1 patients shows high levels of instability with frequent increases in length from one generation to the next. However, although general observations can be drawn by studying individual transmissions through pedigree analysis, there is clearly considerable variation in transmissions, so accurate predictions based on the allele size of the parent cannot be made. In order to provide a greater understanding of germline transmission, we have analysed six sperm samples from DM 1 males by detailed SP-PCR analysis. All of these samples revealed very high levels of mosaicism for the expanded allele, with mutation rates essentially $100 \%$ per gamete. All samples showed twotailed distributions with a lower tail extending back down into the normal size range, consistent with a germline specific mutational path way that generates a low but detectablefraction of reversions, in good agreement with the rate of reversions observed in DM 1 pedigrees. ${ }^{21-23}$ In addition, the threshold for gross instability appears to be much lower in the male germline than in the soma. These data confirm that the male germline mutational pathway is very different from that observed in somatic tissues where such revertant alleles are observed only very rarely. ${ }^{14}$ The reasons for this are currently unclear, since it is not yet known at what stage(s) of spermatogenesis the mutations arise. Although meiosis is an obvious candidate, there is little direct evidence to confirm this other than the correlation of one of the observed reversions with a complex gene conversion event. ${ }^{22}$ Nonetheless, given the high levels of somatic instability observed for large expansions, it seems likely that any meiotic effect would be superimposed upon a premeiotic effect. One factor

Table 1 Allele diversity in the blood and sperm of DMI males as determined using small pool PCR analysis

\begin{tabular}{|c|c|c|c|c|c|c|}
\hline Family & I & II & III & IV & $\mathrm{V}$ & $\mathrm{VI}$ \\
\hline Male & $\mathrm{DM}-1$ & $\mathrm{DM}-2$ & DM-3 & $\mathrm{DM}-4$ & DM-7 & DM-9 \\
\hline Age at sampling (years) & 28 & 36 & 31 & 38 & 25 & 41 \\
\hline Average expanded allele size in blood (repeats) & 240 & 210 & 300 & 630 & 700 & 90 \\
\hline Estimated progenitor allele size (repeats) ${ }^{a}$ & 150 & 150 & 210 & 210 & 390 & 90 \\
\hline Average expanded allele size in sperm (repeats) & 270 & 240 & 240 & 450 & 660 & 800 \\
\hline $\begin{array}{l}\text { Average germline length change of expanded allele } \\
\text { (repeats per sperm) }{ }^{\mathrm{a}}\end{array}$ & +120 & +90 & +30 & +240 & +270 & +710 \\
\hline $\begin{array}{l}\% \text { contractions of the expanded allele in sperm } \\
\text { relative to the progenitor allele }{ }^{a}\end{array}$ & 3 & 1 & 18 & 3 & 19 & 1 \\
\hline
\end{tabular}

aThe progenitor allele length, and hence the average length change per sperm and contraction frequencies, were estimated from the lower boundary of the distribution observed in the somatic DNA (see text for details). 

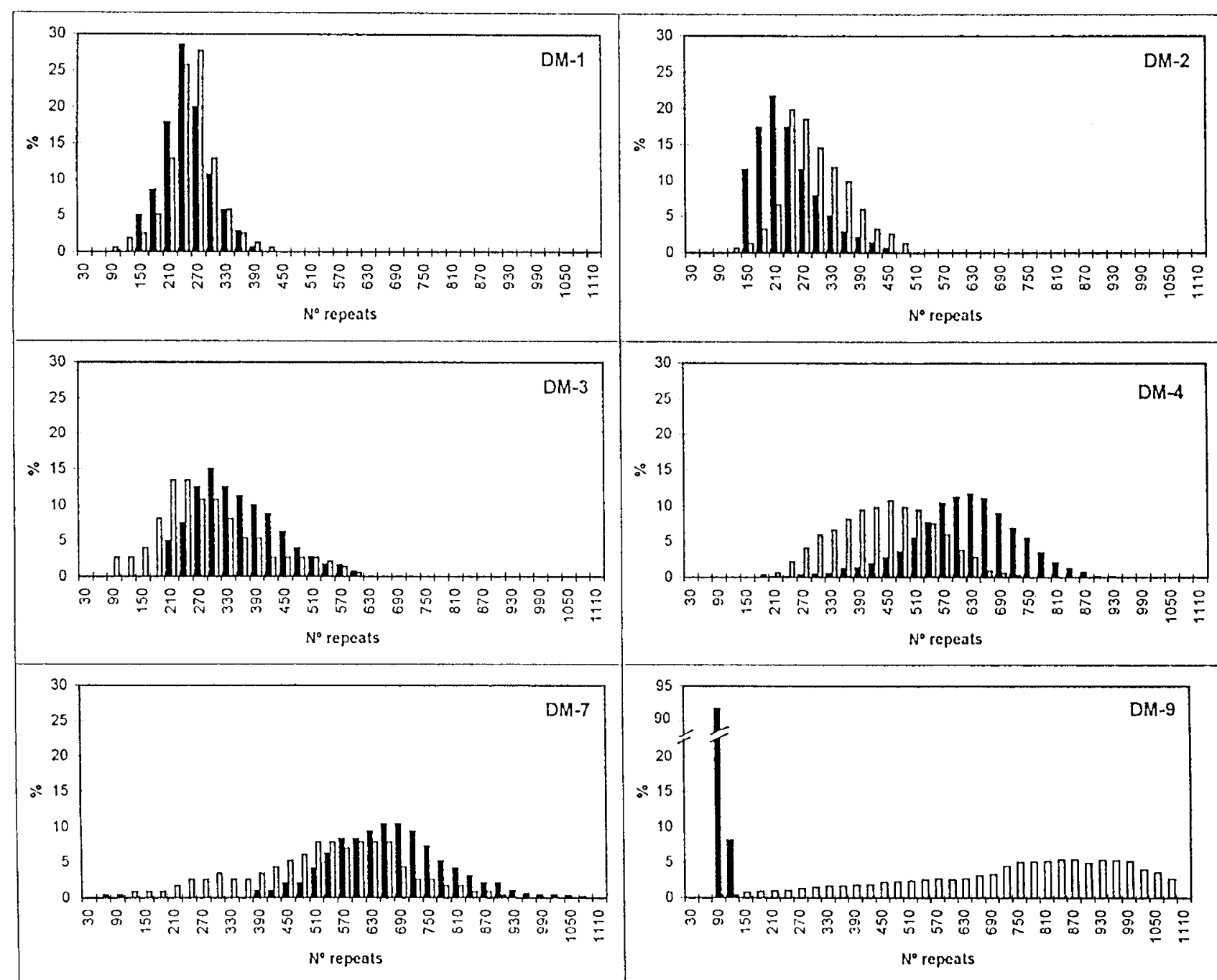

Figure 2 Distribution of variant DM 1 CTG repeat expanded alleles in the blood and sperm of six myotonic dystrophy males. For each individual large numbers of individually amplified expanded alleles were sized from blood and sperm DNA and grouped into 30 repeat size classes. The graphs demonstrate the percentage of expanded alleles falling into the mutant size groups shown. Black bars correspond to blood DNA, shaded bars correspond to sperm DNA.

which is presently unclear is whether male germline instability is also age dependent. So far only one sperm sample has been analysed from any one patient and an obvious age effect in independent samples is not apparent and paternal age effects in DM 1 transmission have not been reported.

Since we know that the DM 1 repeat is highly unstable in somatic tissues, some account of somatic mosaicism needs to be made in attempting to interpret intergenerational transmissions. Small pool-PCR (SP-PCR) analysis of somatic mosaicism in DM1 adults usually reveals highly characteristic distributions, demonstrating very high levels of repeat variability, positively skewed towards expansions and with a sharp individual specific lower boundary conserved between different tissues. ${ }^{14,16,17}$ Since mammalian somatic tissues arise from a large number of precursor cells from an even larger pool of homogeneously mixed cells in the developing embryo, ${ }^{24}$ a lack of significant mosaicism in the blood DNA of DM 1 newborns ${ }^{16,17}$ indicates that the DM 1 repeat does not usually undergo significant length changes prior to organogenesis. Thus, the lower boundary observed in somatic distributions is the best available estimate for the inherited progenitor al lele size (ie the single allele contained within the gamete which forms the zygote and from which all other tissues in the developing embryo and adult were thus originally derived). ${ }^{14}$

Although the average allele size is always larger in muscle compared with blood, the average allele size in muscle DNA shows a worse correlation with disease severity and age of on set. ${ }^{11,12,14,25}$ Thus, since blood is apparently the most stable tissue and closest to the inherited progenitor allele, it seems 
A

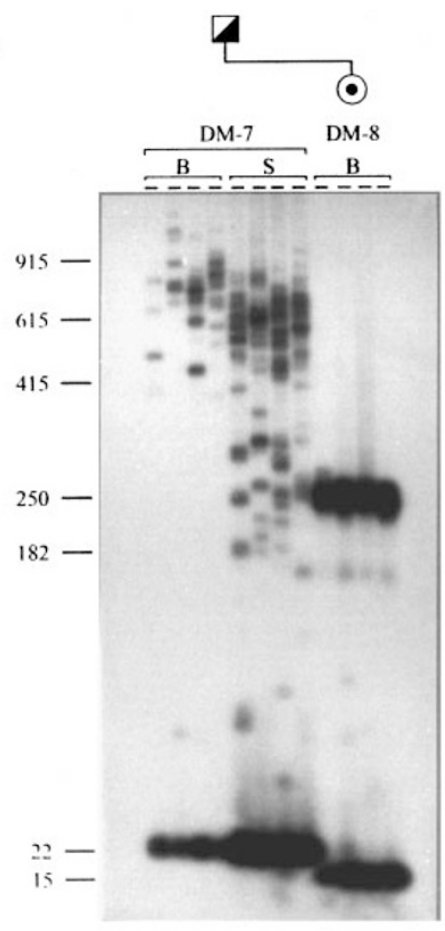

FAMILY-V
B

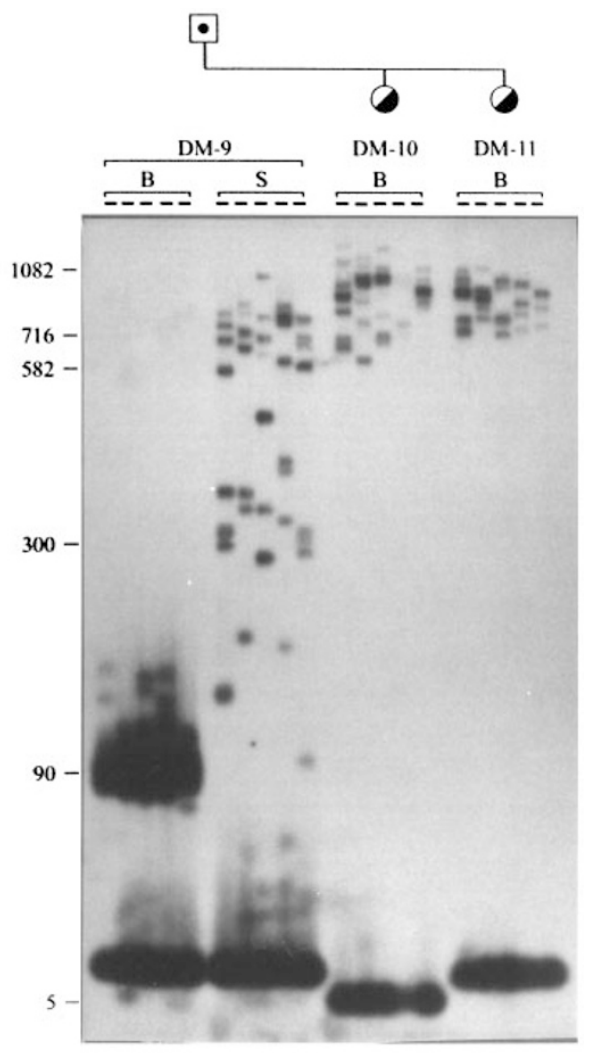

FAMILY-VI

Figure 3 Small pool-PCR comparison of DM 1 CTG repeat alleles in the father's blood and sperm DNA with his children's blood DNA. Shown are representative SP-PCR analyses from families $V(A)$ and $V I(B)$ for the fathers' blood and sperm DNA and their children's blood DNA. For each sample five reactions each containing approximately 10-40 amplifiable expanded molecules are shown.

$\mathrm{B}=$ blood, $\mathrm{S}=$ sperm. The scale shows the position of the molecular weight markers converted into the number of repeats.

Table 2 Allele diversity in the offspring of DM1 males as determined using small pool PCR analysis

\begin{tabular}{|c|c|c|c|c|c|c|}
\hline Family & I & II & III & IV & V & $\mathrm{VI}$ \\
\hline Father & DM-1 & DM-2 & DM-3 & DM-4 & DM-7 & $\overline{D M}-9$ \\
\hline First conception & - & - & - & DM-5 & DM-8 & DM-10 \\
\hline Age at sampling (years) & PND & PND & PND & 10 & 1 & 14 \\
\hline Average expanded allele size (repeats) & 230 & 170 & 330 & 330 & 250 & 1100 \\
\hline Estimated progenitor allele size (repeats) ${ }^{a}$ & 230 & 170 & 330 & 300 & 250 & 600 \\
\hline Estimated inherited germline change (repeats) ${ }^{a}$ & +80 & +20 & +120 & +90 & -140 & +510 \\
\hline Second conception & - & - & - & DM-6 & - & DM-11 \\
\hline Age at sampling (years) & PND & - & PND & 8 & - & 8 \\
\hline Average expanded allele size (repeats) & 230 & - & 330 & 330 & - & 1000 \\
\hline Estimated progenitor allele size (repeats) ${ }^{a}$ & 230 & - & 330 & 310 & - & 800 \\
\hline Estimated inherited germline change (repeats) ${ }^{a}$ & +80 & - & +120 & +100 & - & +710 \\
\hline
\end{tabular}

The progenitor allele lengths, and hence the inherited germline change, was estimated from the lower boundary of the distribution observed in the somatic DNA (see text for details); PND = prenatal diagnosis.

reasonable to assume that the progenitor allele is the major indicator of likely disease severity. Following on, the major indicator of relative disease severity from one generation to the next, will be the difference in length between the progenitor allele inherited by the parent and the progenitor allele inherited by the child ${ }^{14}$ and this difference is the true 
germline change. Estimation of the progenitor allele length in the father and his offspring using SP-PCR in familiesI, II and III, suggests that in fact all five of these transmissions were most likely small length change expansions and would probably have resulted in clinical anticipation. SP-PCR analysis of the DM-4 blood DNA revealed a broad normal distribution, without a clearly defined lower boundary, reflecting significant somatic instability in which all alleles may have expanded beyond the size inherited in the zygote. In such a case it is not possible to predict accurately the progenitor allele length, other than to suggest that the currently observed diffuse lower boundary represent the best estimate for his inherited allele size. The allele sizes inherited by his offspring are larger than the estimated size of the fathers inherited allele size suggesting that these transmissions also likely represent true germline expansions. The re-interpretation of some of these cases as pseudocontractions (ie true expansions masked by age-dependent somatic expansion in the father) provides an obvious resolution to the paradox raised by Ashizawa et al who demonstrated an excess of apparent contractions, particularly during paternal transmission, relative to the paucity of documented cases of a reduction in disease severity from one generation to the next. $^{10}$

Male DM-7 represents the most likely exampleyet reported of a true germline contraction at the DM 1 locus. It is notable that this man had the largest allele in his somatic DNA consistent with the association of apparent contractions with larger measured allele size in the parent. ${ }^{10}$ Similarly, the high proportion of sperm from this man containing contractions (about $19 \%$ ) and in DM-3 (18\%), is consistent with the previously observed clustering of apparent contractions within sibships. ${ }^{10}$

The SP-PCR analysis of blood DNA of DM-9 male revealed a relatively low level of somatic mosaicism and a modal allele size of about 90 repeats. In stark contrast, his sperm DNA showed the widest distribution we have yet observed with alleles ranging from within the high end of the normal size range all the way up to alleles in excess of 1200 repeats, with a mean allele size for the expanded allele in his sperm DNA of 800 repeats. In five of the DM 1 sperm samples analysed here and in other samples analysed previously ${ }^{13,14}$ alleles larger than $700-800$ repeats were very rare. Indeed, it has been previously suggested that there exist a selection limit of about 1000 repeats during spermatogenesis based on a failure to detect such alleles in a number of DM sperm samples. ${ }^{13}$ This is the first time that such large alleles have been observed directly in the germline. In general, the most severe congenital form of DM 1 is associated with female transmission, ${ }^{8,9}$ although a few cases of paternally transmitted CDM 1 have been reported. ${ }^{26-29}$ It seems reasonable to assume that the other men previously identified as transmitting CDM 1 also contained similar large expansions in their sperm. We currently do not know why the expanded allele in this subset of DM 1 males should be prone to such large expansions.
These data provide further support for the hypothesis that germl ine instability at the DM 1 locus is influenced by factors in addition to purely repeat length, possibly trans-acting genetic modifiers of DNA metabolism. Regardless, it is clear that in general males do not transmit as large expansions as do femal es. Whether this reflects some sex-specific difference in the mutational pathway or somelevel of selection either in the spermatogenic cells directly or their support cells remains unclear.

In all of the cases we have examined the, data are consistent with the transmission of alleles that are still present in the father's sperm, suggesting that the main factor affecting intergenerational differences are germline in origin. The average allele size in the sperm was not, however, obviously correlated with the progenitor allele length or the age of the individual, indicating that other individual specific factors are important in modifying repeat stability. Thus, it is not possible to predict accurately the likely dynamics of the repeat in the germline of other DM 1 males. Nonetheless, this study indicates that the direct single molecule analyses of the sperm DNA of individual DM 1 males can be used effectively to predict the range of allele lengths that could be inherited by their offspring. As indicated by several of the cases described here, it is quite possible for germline expansions to be masked by somatic expansion in the father and present as apparent intergenerational contractions. Measuring the intergenerational dynamics of the DM 1 CTG repeat is clearly confounded by the confusing interplay between age-dependent somatic mosaicism and germline instability. Thus, without a greater understanding of the dynamics of the somatic expansion pathway, extreme caution should be used in interpreting intergenerational transmissions measured by comparing average allele lengths in the blood DNA of parent and offspring.

\section{Acknowledgements}

We are indebted to the patients for their co-operation in performing this study and should like to thank Keith Johnson, Peggy Shelbourne and their groups, and all the members of our groups for helpful discussions with this work. This work was supported by a grant (98/1299) from the Fondo de Investigaciones de la Seguridad Social, Ministerio de Sanidad y Consumo, Spain and from Maratò TV 3 (98/0910). DGM is a Lister Institute Research Fellow and was supported by a grant from the Muscular Dystrophy Association (USA). The protocol has been revised and approved by the Ethical Committee of the Hospital de la Santa Creu i Sant Pau.

\section{References}

1 Harper PS: Myotonic dystrophy, 2nd edn. WB Saunders Co: London, 1989.

2 Brook JD, McCurrach ME, Harley HG et al: Molecular basis of myotonic dystrophy: expansion of a trinucleotide (CTG) repeat at the $3^{\prime}$ end of a transcript encoding a protein kinase family member. Cell 1992; 68: 799-808.

3 Buxton J, Shelbourne P, Davies J et al: Detection of an unstable fragment of DNA specific to individuals with myotonic dystrophy. Nature 1992; 355: 547-548. 
4 Ranum LP, Rasmussen PF, Benzow KA, Koob MD, Day JW: Genetic mapping of a second myotonic dystrophy locus. Nat Genet 1998; 19: 196-198.

5 Ricker K, Grimm T, Koch MC et al: Linkage of proximal myotonic myopathy to chromosome3q. Neurology 1999; 52: 170-171.

6 Barceló JM, Mahadevan MS, Tsilfidis C, Mackenzie AE, Korneluk RG: Intergenerational stability of the myotonic dystrophy protomutation. Hum Mol Genet 1993; 2: 705-709.

7 Brunner HG, Bruggenwirth HT, Nillsen W et al: Influence of sex of the transmitting parent as well as of parental allele size on the CTG expansion in myotonic dystrophy (DM). Am J Hum Genet 1993; 53: 1016-1023.

8 Lavedan C, Hofmann-Radvanyi H, Shelbourne P et al: Myotonic dystrophy: size and sex-dependent dynamics of CTG meiotic instability, somatic mosaicism. Am J Hum Genet 1993; 52: 875-883.

9 Tsilfidis C, MacKenzie AE, Mettler G, Barcelo J, Korneluk RG: Correlation between CTG trinucleotide repeat length and frequency of severe congenital myotonic dystrophy. N at Genet 1992; 1: 192-195.

10 Ashizawa T, Anvret M, Baiget $M$ et al: Characteristics of intergenerational contractions of the CTG repeat in myotonic dystrophy. Am J Hum Genet 1994; 54: 414-423.

11 Anvret M, Ahlberg G, Grandell U, Hedberg B, Johnson K, Edstrom $\mathrm{L}$ : Larger expansions of the CTG repeat in muscle compared to lymphocytes from patients with myotonic dystrophy. Hum Mol Genet 1993; 2: 1397-1400.

12 Ashizawa T, Dubel JR, Harati Y: Somatic instability of CTG repeat in myotonic dystrophy. Neurology 1993; 43: 2674-2678.

13 Jansen G, Willems P, Coerwinkel M et al: Gonosomal mosaicism in myotonic dystrophy patients: Involvement of mitotic events in (CTG)n variation and selection against extreme expansion in sperm. Am J Hum Genet 1994; 54: 575-585.

14 Monckton DG, Wong L-JC, Ashizawa T, Caskey CT: Somatic mosaicism, germline expansions, germline reversions and intergenerational reductions in myotonic dystrophy males: small pool PCR analyses. Hum Mol Genet 1995; 4: 1-8.

15 Martorell L, Martinez JM, Carey $N$, Johnson $K$, Baiget $M$ : Comparison of CTG repeat length expansion and clinical progression of myotonic dystrophy over a five year period. J Med Genet 1995; 32: 593-596.
16 Martorell L, Monckton DG, Gamez J et al: Progression of somatic CTG repeat length heterogeneity in the blood cells of myotonic dystrophy patients. Hum Mol Genet 1998; 7: 307-312.

17 Wong L-JC, Ashizawa T, Monckton DG, Caskey CT, Richards CS: Somatic heterogeneity of the CTG repeat in myotonic dystrophy in age and size dependent. Am J Hum Genet 1995; 56: 114-122.

18 Jeffreys AJ, Tamaki K, MacLeod A, Monckton DG, Neil DL, Armour JAL: Complex gene conversion events in germline mutation at human minisatellites. Nat Genet 1994; 6: 136-145.

19 Maniatis T, Firtsch EF, Sambrook J: A simple salting out procedure for extracting DNA from human nucleated cells. Nucleic Acids Res 1988; 16: 1215.

20 Cobo A, Martinez JM, Martorell L, Baiget M, Johnson K: Molecular diagnosis of homozygous myotonic dystrophy in two asymptomatic sisters. Hum Mol Genet 1993; 2: 711-715.

21 Brunner HG, Jansen G, Nillsen W et al: Reverse mutation in myotonic dystrophy. N Eng J Med 1993; 328: 476-480.

22 O'Hoy KL, Tsilfidis C, Mahadevan MS et al: Reduction in size of the myotonic dystrophy trinucleotide repeat mutation during transmission. Science 1993; 259: 809-812.

23 Shelbourne $P$, Windquist $R$, Kunert $E$ et al: Unstable DNA may be responsible for the incomplete penetrance of the myotonic dystrophy phenotype. Hum Mol Genet 1992; 1: 467-473.

24 Rossant J: Cell lineage analysis in mammalian embryogenesis. Curr Top Dev Biol 1987; 23: 115-146.

25 Thornton CA, Johnson KJ, Moxley RT: Myotonic dystrophy patients have larger CTG expansions in skeletal muscle than in leukocytes. Ann Neurol 1994; 35: 104-107.

26 Bergoffen J, Kant J, Sladky J, McDonald-McGinn D, Zackai EH, Fischbeck KH: Paternal transmission of congenital myotonic dystrophy. J Med Genet 1994; 31: 518-520.

27 Die Smulders CEM, Smeets HJM, Loots W et al: Paternal transmission of congenital myotonic dystrophy. J Med Genet 1997; 34: 930-933.

28 Nakagawa M, Yamada $\mathrm{H}$, Higuchi I et al: A case of paternally inherited myotonic dystrophy. J Med Genet 1994; 31: 397-400.

29 Ohya K, Tachi N, Chiba S et al: Congenital myotonic dystrophy transmitted from an asymptomatic father with a DM specific gene. Neurology 1994; 44: 1958-1960. 